\title{
EL COMPORTAMIENTO DEL CONSUMIDOR EN LA GESTIÓN COMERCIAL DE DESTINOS TURÍSTICOS SUCRE-SAN VICENTE. UN ACERCAMIENTO DESDE LAS CIENCIAS SOCIALES, ECUADOR 2017
}

THE BEHAVIOR OF THE CONSUMER IN THE COMMERCIAL MANAGEMENT OF TOURIST DESTINATIONS SUCRE-SAN VICENTE. AN APPROACH FROM THE SOCIAL SCIENCES, ECUADOR 2017

Frank Ángel Lemoine Quintero

Docente titular de la Universidad Laica Eloy Alfaro de Manabí, Extensión Bahía de Caráquez, Ecuador.

E-mail: fangel64@gmail.com ORCID: https://orcid.org/0000-0001-8885-8498

María Gabriela Montesdeoca Calderón

Docente Titular de la Escuela Superior Politécnica Agropecuaria de Manabí “ Manuel Félix López”, Ecuador.

E-mail: magymontesdeoca@gmail.com ORCID: https://orcid.org/0000-0003-4752-260X

Lilia Moncerrate Villacís Zambrano

Docente tiempo completo de la carrera de Ciencia de la Educación y Marketing de la Universidad Laica Eloy Alfaro de

Manabí, Extensión Bahía de Caráquez. Ecuador.

E-mail: liviza@hotmail.com ORCID: https://orcid.org/0000-0002-2888-6363

Norma Rafaela Hernández Rodríguez

Docente tiempo completo de la Universidad de Oriente. Investigadora acreditada de la facultad de Ciencias Económicas y

Empresariales. Santiago de Cuba, Cuba.

E-mail: norma@uo.edu.cu ORCID: https://orcid.org/0000-0002-2086-2236

Recepción: 19/10/2018 Aceptación: 22/07/2019 Publicación: 24/02/2020

Gitación sugerida:

Lemoine, F. A., Montesdeoca, M. G., Villacís, L. M., y Hernández, N. R. (2020). El comportamiento del consumidor en la gestión comercial de destinos turísticos Sucre-San Vicente. Un acercamiento desde las ciencias sociales, Ecuador 2017.3C Empresa. Investigación y pensamiento crítico, 9(1), 17-39. doi: http://doi.org/10.17993/3cemp.2020.090141.17-39 


\section{RESUMEN}

La investigación fundamenta su estudio en analizar el comportamiento del consumidor en la gestión del mismo en su entorno sociocultural y medioambiental. A través del método analítico se determinó que la actividad turística tiene un impacto importante en el medio económico debido a su efecto multiplicador que concibe esta actividad la cual brinda ingresos a las comunidades ubicadas en cada uno de los cantones generando servicios para todas las empresas y microempresas que operan en las localidades. Se estudia las contradicciones epistemológicas, políticas, económicas, culturales y ambientales ante la problemática social en sus dimensiones y su aporte a la gestión del destino turístico en la zona objeto estudio. Se propone adoptar un enfoque integrado, que tenga en cuenta las dimensiones económica, social y ambiental para lograr una gestión comercial de destinos turísticos eficiente y eficaz que responda a las necesidades de los consumidores.

\section{PALABRAS CLAVE}

Consumidor, Comportamiento, Destino, Dimensiones, Enfoque integrado. 


\section{ABSTRACT}

The research bases its study on analyzing consumer behavior in the commercial management of the tourist destination of the Sucre cantons -San Vicente and his approach to the social sciences. The review of bibliographic sources allowed to conceptualize the consumer through the characteristics and guidelines of action in order to verify the action of the same in their sociocultural and environmental environment. Through the analytical method it was determined that tourism activity has a significant impact on the economic environment due to its multiplying effect that conceives this activity which provides income to the communities located in each of the cantons, generating services for all companies and micro businesses that operate in the localities. The epistemological, political, economic, cultural and environmental contradictions are studied in the face of the social problems in their dimensions and their contribution to the management of the tourist destination in the study area. It is proposed to adopt an integrated approach that considers the economic, social and environmental dimensions to achieve a commercial management of efficient and effective tourism destinations that meets the needs of consumers.

\section{KEYWORDS}

Consumer, Behavior, Destination, Dimensions, Integrated approach. 


\section{INTRODUCCIÓN}

El turismo comprende las actividades que realizan las personas (turistas) durante sus viajes y estancias en lugares distintos al de su entorno habitual, por un periodo de tiempo consecutivo inferior a un año, con fines de ocio, por negocios y otros motivos (Ghinis, 2009).

En el siglo XVI hasta mediados del siglo XIX se establecen las bases del "turismo moderno", ya que durante este periodo tiene su origen el denominado Gran Tour del que posteriormente se deriva el termino turismo, que consistía en enviar a los jóvenes de la nobleza y de la clase media inglesa a viajar al continente con el fin de complementar sus conocimientos, aprender costumbre de esos países y ganar más experiencia personal. El Grand Tour es un viaje motivado por la necesidad de instrucción de estos jóvenes aristócratas que en un futuro habrán de gobernar su país.

Estos desplazamientos se diferencian por su propósito de otros tipos de viajes motivados por guerras, movimientos migratorios, conquista, comercio, entre otros. El turismo radica en los viajes y permanencia que hacen las personas en sitios diferentes de su ambiente frecuente, viajes realizados por diversión, finalidad mercantil o profesional y otros semejantes, mientras la estancia en el lugar sea eventual. Hoy el turismo es una de las industrias más importantes a nivel mundial y promociona viajes de todo tipo, influye a que la actividad turística proviene de tiempos antiguos; abarca una gran diversidad de sectores económicos y disciplinas académicas.

El producto turístico es el conjunto de atributos tangibles e intangibles reunidos en una forma identificable, con un nombre que lo describe, que genera utilidades o beneficios al cliente y que el mismo puede aceptar como satisfactorio de una o más necesidades. Puede definirse, como "todo aquello que se pueda ofrecer a un mercado turístico para que se adquiera, se use, se consuma o se le preste atención, con el objetivo de satisfacer un deseo o necesidad” (Mármol, García y Ojeda, 2016).

El desarrollo alcanzado por la actividad turística en los últimos años ha permitido que se maduren ciertas ideas en cuanto a elaborar términos específicos que permitan definir las diferentes actividades 
que en este sector se realizan. El producto turístico debe estar conformado por aquel producto de uso exclusivo de los turistas que es todo plan programa de viaje de ida y vuelta. Tiene su principal insumo en los atractivos, en torno del cual giran una serie de elementos que permiten que se desarrolle la actividad turística en un espacio determinado.

El producto turístico es un conjunto de atractivos, servicios, equipamientos, infraestructura y organizaciones que satisfacen un deseo o necesidad de los consumidores turísticos. Dicho producto es brindado en el sitio (destino) turístico y consumido en el lugar del servicio, lo cual presume un desplazamiento del consumidor desde un lugar de origen a uno de destino y su posterior retorno al sitio de partida.

El sector turístico ha experimentado (aunque de una manera más tardía) una evolución marcada de la orientación de su marketing, ya que durante décadas su único objetivo era producir y vender. La elaboración de paquetes turísticos estandarizados, de idéntica composición y venta masiva, permitía a las personas, olvidándose por completo de cubrir las expectativas de la muy diversa tipología de clientes.

En la actualidad existen mercados dinámicos y en rápida evolución, que requieren; se conozca, todo lo que sea posible acerca de los consumidores. Esta diversidad del mercado impulsa a los mercadólogos a establecer segmentos de mercado para satisfacer las necesidades específicas de sus consumidores. La clave de la supervivencia, la rentabilidad y el crecimiento de una empresa en un mercado altamente competitivo, es su capacidad para identificar y satisfacer necesidades.

El comportamiento del consumidor resulta el arma más eficaz para enfocar los esfuerzos de una empresa en la satisfacción de necesidades específicas, las empresas deben tomar en cuenta que el consumidor es un individuo con fuerzas psicológicas y con un contexto social y cultural tan diverso que debe ser estudiado de forma permanente.

Según el Diccionario Manual de la Lengua Española de la Editorial Larousse, Edición 2007, la satisfacción es un sentimiento de bienestar o placer que se tiene cuando se ha colmado un deseo o 
cubierto una necesidad, entonces se podría decir que una persona puede estar enteramente satisfecha cuando sus deseos o expectativas de un servicio o producto estén cubiertas por la calidad del mismo.

En el Ecuador el turismo ha experimentado un crecimiento sostenible en los últimos años, sobre todo en lo relacionado al turismo internacional, no obstante, aún existen falencias que atentan contra el desarrollo del sector turístico y al cambio de la matriz productiva, entre las que se pueden citar:

- Bajos porcentajes de ocupación de la planta hotelera disponible.

- Marcada estacionalidad.

- Bajos ingresos por turista.

- Escaso enfoque hacia el cliente lo que afecta la calidad del servicio y la satisfacción de los turistas.

- Deficiente gestión comercial en los destinos turísticos de la zona.

Así, constituye objetivo de este trabajo analizar las contracciones sociales que afectan el comportamiento del consumidor y que frenan el desarrollo de los destinos turísticos en Ecuador.

\section{DESARROLLO}

\subsection{PRINCIPALES CONCEPCIONES ACERCA DE LOS CONSUMIDORES}

El concepto de comportamiento del consumidor se deriva de la noción de marketing, la cual empezó a ser reconocida a finales de la década de 1950.

El progreso que ha tenido el marketing se manifiesta mediante las distintas definiciones que lo han llevado a nutrirse tales como: actividad, proceso, función, sistema, enfoque, disciplina e inclusive, en otro entorno como ciencia de gestión normativa. Referente al entorno lo conceptual tiene su explicación 
debido a las dimensiones del marketing principalmente al relacional con la finalidad de analizar y darle seguimiento de las necesidades del individuo y de las organizaciones para complementar sus expectativas. Situación que haría dudar de sus conocimientos y pertenencia al entorno de la misma, por dos argumentos elementales: el modelo es el característico que permitirá entender la definición del marketing, y el periodo obtiene asociar con herramientas y proceso de exploración y formación.

En esa época las empresas se dieron cuenta de la facilidad con la que se vendían los productos o servicios que contaban con información previa acerca de las necesidades específicas que iban a satisfacer en el consumidor. Esto les permitía eliminar prácticas inusuales como producir para posteriormente vender, sin tomar en cuenta si eran o no del agrado de los compradores o usuarios. En la actualidad las organizaciones más exitosas del mundo han alcanzado un nivel de satisfacción de los consumidores, transformando la organización entera para servirles y permanecer cerca de ellos. Para alcanzar este objetivo, estas empresas han generado un enfoque para el consumidor, en donde han determinado en primer lugar qué quieren para poder diseñar, producir y comercializar productos o servicios con la más alta calidad y a precios razonables.

Realizar este tipo de enfoques, se fundamenta en el aumento de las investigaciones acerca de los consumidores y la importancia de contar con información detallada sobre: qué, dónde, cuánto, cuándo y por qué compran y/o consumen.

Paul y Olson (2006) afirman que "El comportamiento del consumidor abarca los pensamientos y sentimientos que experimentan las personas, así como las acciones que emprenden, en los procesos de consumo".

De la anterior definición, se puede deducir que:

- Los sentimientos, pensamientos y acciones de cada consumidor se modifican a cada momento gracias a que su medio ambiente está también en constante movimiento, los ciclos de vida de los 
productos son más breves y las empresas se ven en la necesidad de revisar de forma constante sus estrategias de marketing.

- Los sentimientos, pensamientos y acciones de los consumidores tienen importantes interacciones con su medio ambiente, proporcionando información a los mercadólogo sobre las preferencias de algunos productos o servicios y el porqué de esas preferencias.

- El intercambio entre los seres humanos es algo inevitable, el comportamiento de los consumidores consiste en que las personas entreguen dinero a otras para obtener productos y servicios, que satisfagan necesidades.

\subsection{FACTORES QUE INFLUYEN EN EL COMPORTAMIENTO DEL CONSUMIDOR}

En las compras de los consumidores influyen marcadamente factores externos que repercuten en la conducta grupal como lo son características culturales, económicas, sociales, personales y psicológicas, así como factores internos que determinan el comportamiento individual tales como el aprendizaje, la personalidad, motivación, memoria y actitudes.

Tabla 1. Tipos de consumidor o clientes.

\begin{tabular}{|c|c|c|}
\hline $\begin{array}{l}\text { Tipología del } \\
\text { cliente }\end{array}$ & Características & Pautas de actuación \\
\hline Racional & $\begin{array}{l}\text { - Sabe lo que quiere y necesita. } \\
\text { - Es concreto y conciso en sus demandas y } \\
\text { opiniones. } \\
\text { - Pide información exacta. }\end{array}$ & $\begin{array}{l}\text { - Demostrar seriedad e interés. } \\
\text { - Demostrar seguridad y profesionalidad. } \\
\text { - Ofrecer información precisa y completa. }\end{array}$ \\
\hline Reservado & $\begin{array}{l}\text { - Evita mirar a los ojos. } \\
\text { - Procura mantener distancia con el vendedor. } \\
\text { - } \text { No exterioriza sus intereses ni opiniones. } \\
\text { - Busca información completa. } \\
\text { - Necesita tiempo para valorar y decidir. }\end{array}$ & $\begin{array}{l}\text { - Hacer preguntas de respuesta fácil. } \\
\text { - Animarle a que exprese sus demandas y } \\
\text { opiniones. } \\
\text { - Mostrar variedad de productos. } \\
\text { - No interrumpir sus intervenciones. } \\
\text { - Mostrar calma y tranquilidad y no presionar. } \\
\text { - Dejarle tiempo para pensar. } \\
\text { - Atender a otros clientes mientras decide. }\end{array}$ \\
\hline
\end{tabular}




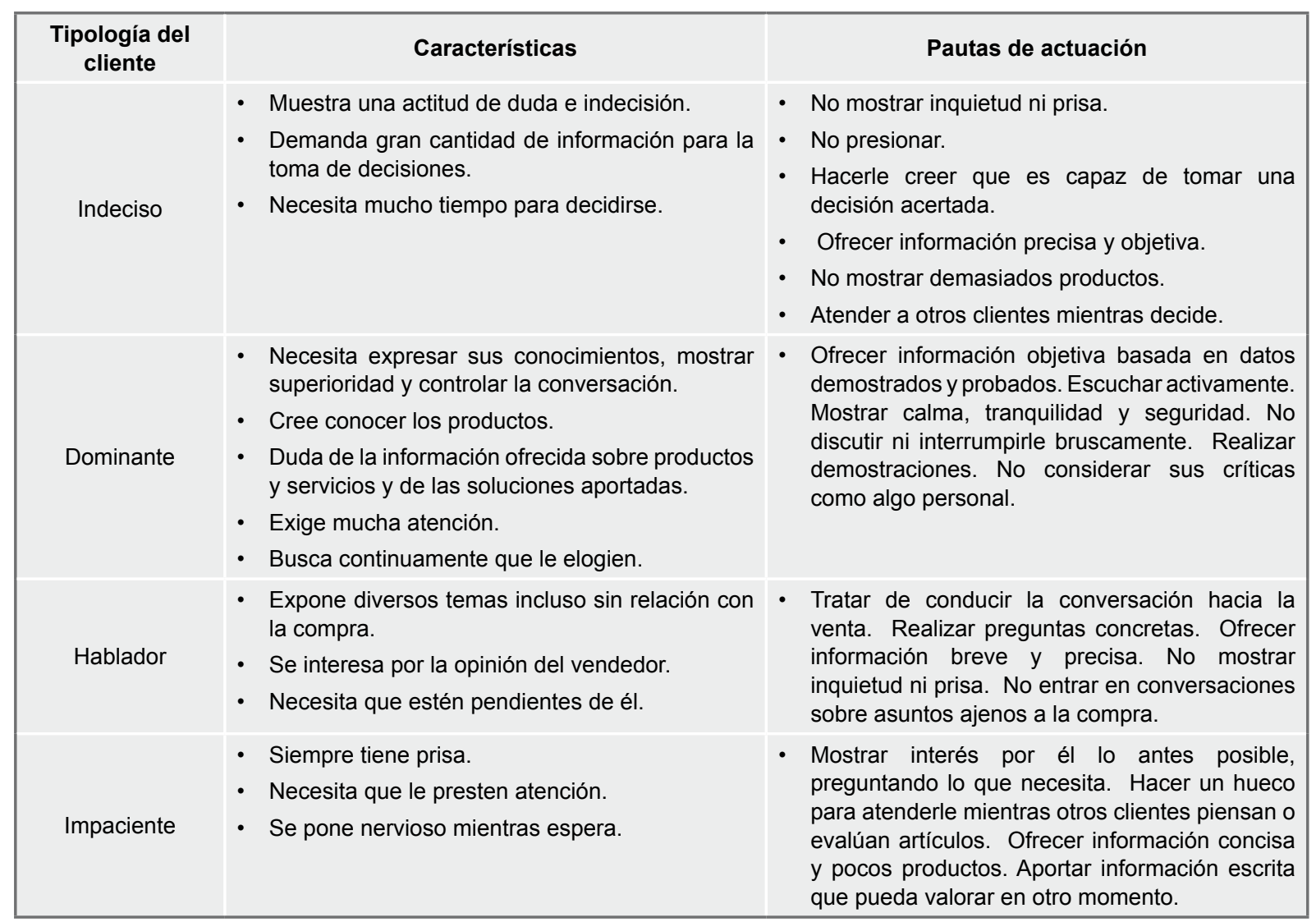

Fuente: elaboración propia.

La tipología de los consumidores no es estática, sino que cada sujeto puede variar su conducta dependiendo del entorno o momento en el cual se encuentren. Por otro lado estas pausas de actuación son solo una guía de trabajo, en ningún momento debemos transformar la atención del cliente, sino tratar de personalizarla y hacer sentir cada cliente de forma única. 
El mercado turístico es el lugar donde confluyen, por un lado, la oferta de productos y servicios turísticos y, por el otro, la demanda de los mismos productos y servicios turísticos. El mercado turístico se encuentra conformado por: La oferta turística, La demanda turística y los operadores turísticos.

Estas tres características del mercado turístico son complementarias para conocer el comportamiento que tiene el consumidor de dicho servicio, además considero que la oferta turística es la que engloba a las otras características ya que esta es el conjunto de elementos que conforma al producto turístico que es manejado por los operadores turísticos que a su vez influyen para tener esa demanda turística. Es importante el análisis del comportamiento del consumidor en su entorno tanto económico, como social cultural y medioambiental para verificar como inciden cada uno de ellos en esa conducta del consumidor, por lo que lo reflejamos a continuación en el siguiente cuadro:

Tabla 2. Impacto genera el turismo en el medio.

\begin{tabular}{|c|c|c|}
\hline Impacto económico & Impacto sociocultural & Impacto medioambiental \\
\hline $\begin{array}{c}\text { Creación de empleo, la entrada de } \\
\text { divisas que ayudan a equilibrar la } \\
\text { balanza de pagos, el aumento de los } \\
\text { ingresos públicos y el fomento de la } \\
\text { actividad empresarial. }\end{array}$ & $\begin{array}{c}\text { Las tradiciones, costumbres y } \\
\text { patrimonio histórico. }\end{array}$ & $\begin{array}{c}\text { Los problemas de contaminación, } \\
\text { depredación, deforestación, etc. }\end{array}$ \\
\hline
\end{tabular}

Fuente: elaboración propia.

La actividad turística tiene un impacto importante en el medio económico debido a su efecto multiplicador que genera esta actividad la cual brinda ingresos al cantón para todas las empresas y microempresas que operan en la localidad. Las tradiciones costumbres y patrimonio histórico que posee el cantón junto con sus parroquias es uno de los referentes más atractivos para atraer a nuestros visitantes. Por otro lado no se puede dejar atrás el impacto ambiental, ya que es un factor muy importante para lo cual hay que tomar medias para la conservación y mejoramiento de la calidad ambiental, en ese sentido, el turismo puede y debe contribuir con la revalorización del entorno natural de las zonas en las que desarrolla su actividad, pues un entorno bien preservado ayuda a elevar la calidad de la actividad turística. 
El destino, como ámbito donde ocurre la mayoría de las actividades de producción y consumo turístico y tiene lugar gran parte de los efectos ambientales, sociales, económicos y culturales del turismo; no solo ha carecido de la debida atención que merece, sino también ha sido abordado indistintamente como sinónimo de producto o como el lugar donde se asienta el turismo. Todo ello motiva que aún hoy no cuente con una adecuada definición.

Los destinos conforman realmente un sistema en el espacio, donde se entrecruzan otros dos sistemas más amplios: el turístico y el geográfico, y como tal actúa entre ambos. Por lo cual debe ser interpretado, planificado y gestionado, atendiendo tanto a sus elementos individuales (propios) como también a las interacciones que entre esos y otros elementos (incorporados) se producen.

Algunos autores, al considerar que la razón última del turismo son los servicios que se brindan en un espacio dado (alojamiento, restauración, recreación, etc.) olvidan el hecho de que esto es sólo una parte de la oferta, no fundamental en muchos casos, como tampoco es la motivación principal para el visitante.

De lo expuesto se comprenderá que aun cuando puedan existir diversos criterios sobre el concepto y funcionamiento de los destinos turísticos, éstos siguen siendo una realidad compleja y esencial contentiva de múltiples y variadas relaciones, lo cual demanda para su desenvolvimiento una gestión y planificación que no puede reducirse a un simple ordenamiento territorial, ni tampoco, sólo al estudio del ámbito natural y los impactos que el turismo puede provocar en el mismo.

Al ser el destino turístico el soporte de la oferta, es un espacio concreto y objetivo, de dimensiones y formas variables. A su vez, dicho espacio es percibido a través de valores sociales (gustos, modas, etc.) los cuales imponen su propia representación, y constituyen parte importante de su valor turístico - recreativo, por el cual el espacio se convierte en un símbolo más allá de sus propios atractivos. En resumen, el territorio es además de soporte físico, una parte indisoluble del propio producto a consumir.

Como se apuntó antes, a pesar de la trascendencia de los destinos para el desarrollo del turismo aún no se ha conseguido una interpretación definitiva del concepto. De hecho, en un número de la revista 
TedQual editada por la Organización Mundial del Turismo, se expresaba críticamente lo lamentable de que este "espacio local de la actividad turística cuya importancia es a todas luces innegable, permanezca en una tiniebla relativa desde el punto de vista conceptual y analítico" (OMT, 2002).

Aún hoy el término "destino" mantiene una cierta indefinición de la que se derivan no sólo problemas interpretativos sino también de planificación y gestión. Como resultado, el Grupo de Expertos en Gestión de Destinos de la OMT, en reunión celebrada en Madrid, España, en diciembre de 2002, intentó zanjar el asunto con la siguiente definición: "Un destino turístico local es un espacio físico en el cual un turista está al menos una noche. Incluye productos turísticos tales como servicios de apoyo, atracciones y recursos turísticos que pueden ser consumidos en un recorrido de ida y vuelta el mismo día. Tiene fronteras físicas y administrativas que definen su gestión, e imágenes y percepciones que definen su posicionamiento en el mercado. Los destinos locales incorporan varios agentes entre los cuales se incluye la sociedad local y pueden establecer redes que formen destinos mayores".

Esa definición contiene aspectos discutibles, como el de los límites físicos y administrativos, entre otras cuestiones que aún siguen pendientes. La propia organización ha planteado la necesidad de estudiar los posibles criterios para establecer los límites físicos, una clasificación morfológica y la dinámica de los destinos turísticos.

En esencia el destino es un sistema de relaciones, más que un conjunto de componentes (instalaciones y atractivos). Este modo de abordar el estudio de los destinos turísticos permite identificarlos no como una realidad preexistente (producto o territorio) sino como elementos relacionados que en su dinámica generan una imagen percibida por los visitantes (Rodríguez, 2011; Bigné, Font y Andreu, 2001).

El éxito de un destino turístico se relaciona con la presencia de productos turísticos que se complementen y generen una oferta atractiva para los visitantes, de manera tal que puedan permanecer más tiempo en el lugar sin aburrirse, participando cada día en una experiencia nueva. Por ello, un primer objetivo para un destino turístico es sumar esfuerzos entre las entidades nacionales y locales con las empresas turísticas 
del territorio, para asociarse de cierta manera en la promoción y comercialización de sus productos, en busca de un posicionamiento adecuado en los mercados. Para ello se requiere de una visión estratégica igualmente compartida.

$\mathrm{Al}$ considerar que los territorios donde se establece la actividad turística se constituyen en espacios con determinadas características y condiciones para que esa actividad se desarrolle adecuadamente, es necesario entonces que los mismos sean contemplados y atendidos como una entidad específica. Sin embargo, los territorios donde actúa de modo preferente o exclusivo el turismo no son una empresa. En los destinos turísticos están presentes diversas estructuras empresariales e institucionales que hacen de los mismos un sujeto complejo, dada la interacción de tales formas organizativas. Por tanto, para lograr un desarrollo armónico y que las acciones de cada una de las empresas e instituciones participantes directa o indirectamente en el negocio turístico contribuyan al bienestar de los visitantes y de todo el sistema, es necesaria la existencia de una coordinación efectiva para la conducción de esa peculiar organización.

Es en ese contexto donde adquiere importancia el problema de la gestión. El destino, como sistema integrado por diversos componentes que interactúan en un espacio determinado y cuyo resultado o "salida" es la oferta de servicios, no puede surgir, crecer, funcionar y evolucionar de modo anárquico; por el contrario, requiere de la necesaria coordinación entre los "actores" presentes en ese territorio para poder mostrar al visitante un ambiente donde prime la adecuada fluidez y armonía necesaria en toda operación turística.

Ante tales requerimientos resulta relevante la gestión del destino turístico. Entendiéndose por tal el conjunto de decisiones y acciones que conducen al logro de objetivos previamente definidos.

Esta labor se realiza en función de espacios y tiempos específicos que determinan su organización y modo de actuar. Los territorios que se constituyen en destinos turísticos cuentan con un conglomerado de productos y servicios que se ofertan en un lugar geográfico determinado, por lo cual una tarea fundamental de sus gestores es ocupase de integrar los distintos recursos, bienes y servicios, equipamientos 
e infraestructuras (que afectan directa o indirectamente la actividad turística), de manera que se diseñe una planificación común y se realice una gestión coordinada de todo ese sistema. Ello no puede efectuarse fuera del territorio.

\subsection{COMPORTAMIENTO DEL CONSUMIDOR}

El comportamiento del consumidor, como una disciplina del Marketing se enfoca en la forma que los individuos toman decisiones para gastar sus recursos disponibles (tiempo, dinero y esfuerzo) en artículos relacionados con el consumo.

Para María Fernanda y Ana María (Comportamiento del consumidor, 2011) el comportamiento del consumidor "es el estudio de personas, grupos u organizaciones y los procesos que siguen para seleccionar, conseguir, usar y disponer de productos, servicios, experiencias o ideas para satisfacer necesidades y los impactos que estos procesos tienen en el consumidor".

Así mismo Hawkins, Best, y Coney (2004) y Kotler y Armstrong (2007) en su libro Fundamentos de Marketing nos definen el comportamiento del consumidor como: "el comportamiento de compra de los consumidores finales individuos y hogares que adquieren bienes y servicios para su consumo personal."

\section{INDIGADORES DE SATISFAGGIÓN (INTERGONSULT, s.f.)}

- Índice de Importancia Relativa de los atributos (IIR).

- Índice de desempeño de los atributos de calidad (IDAG).

- Índice de desempeño de las áreas de atributos de calidad (IDAR).

- Índice satisfacción del cliente con calidad percibida (ISCP).

- Índice de satisfacción con el precio percibido (ISPP).

- Índice de satisfacción del cliente (ISG). 
- Grado de fidelidad (GF).

A partir de este índice es posible:

- Determinar el grado de impacto de cada atributo en la satisfacción y fidelidad del cliente.

- Establecer "mapas" o matrices de apoyo a la definición de mejoras en los servicios.

- Definir segmentos de clientes de acuerdo a su grado de fidelidad.

El sistema funciona en base a un diseño de atributos que hacen al servicio agrupados por áreas. Estos atributos deben ser determinados a priori, ya sea en base a la experiencia y conocimiento que se tenga del servicio o a estudios cualitativos (entrevistas o grupos focus) realizados especialmente para definir estos atributos. A modo de ejemplo, aquí se definen las áreas de atributos "clásicas" de un servicio genérico:

- Calidad de los productos.

- Atención al cliente.

- Comunicación con el cliente.

- Imagen de la empresa.

\section{DISCUSIÓN}

\subsection{ANÁLISIS DE LAS CONTRADICCIONES SOCIALES}

En el proceso de evaluación del comportamiento del consumidor en la gestión de destinos turísticos los destinos turísticos se manifiestan las siguientes contradicciones:

\subsection{CONTRADICCIONES EPISTEMOLÓGICAS}

En la bibliografía consultada se constató la existencia de algunas teorías relacionadas con la evaluación del comportamiento del consumidor en la gestión de destinos turísticos. Algunos autores han enfrentado 
el reto de evaluar el comportamiento del consumidor y su influencia en la gestión comercial de destinos turísticos, observándose enfoques diferentes, lo que evidencia una insuficiente unidad metodológica frente al complejo fenómeno de las evaluaciones del comportamiento de los turistas y su dinámica actual.

La complejidad del sector turístico en Ecuador exige que en la evaluación del cliente se adopte un enfoque integrado de las dimensiones de la realidad social, no obstante, en la teoría se observa una tendencia a priorizar el enfoque financiero o el enfoque socioeconómico de la teoría neoclásica y no fundamentalmente los aspectos de expectativas y satisfacción de los mismos.

\subsection{CONTRADICCIONES POLÍTICAS}

Históricamente las actividades económicas fundamentales de Ecuador estaban relacionadas con los servicios, el comercio y otras actividades. Con la Revolución ciudadana se prioriza el desarrollo de la economía y producción nacional, a pesar de ello aunque existen leyes para mejorar la forma de vida de la población, no están generalizados la aplicación de métodos y procedimientos para evaluar el comportamiento del consumidor y lograr la satisfacción del mismo que redundará al final en beneficios económicos.

Una parte importante de la gestión de los destinos está dedicada a las acciones de promoción para atraer a los visitantes. Sin embargo, como ya se ha apuntado, no es menos relevante la preocupación que debe existir por satisfacer las necesidades de los turistas y de la propia comunidad receptora.

De una u otra forma, lo señalado conduce al análisis del papel a desempeñar por los entes públicos y privados en relación con el desarrollo turístico y, particularmente las funciones estatales en cuanto a la orientación, regulación y desarrollo de la actividad turística en el país, así como su interacción con las formas de gestión de los destinos.

La propia complejidad del fenómeno turístico, donde se mezclan cuestiones de carácter sectorial (producto turístico) con lo territorial (soporte de producción y consumo), obligan a contextualizar el 
espacio (físico - geográfico) de conformidad con los procesos que ocurren en el mismo, los cuales son generadores de una impronta específica en lo social, económico y ambiental. De la interpretación y aplicación de esa realidad compleja es que surgen ahora los estudios territoriales del turismo y, como resultado más actual de los mismos, se encuentran los diversos estudios relativos a los destinos y sus implicaciones para el desenvolvimiento del turismo desde el punto de vista espacial.

\subsection{CONTRADICCIONES ECONÓMICAS}

El turismo es frecuentemente identificado como un sector muy promisorio para el desarrollo de los países, representando una oportunidad de diversificación económica. El principal objetivo de la promoción del turismo es acelerar el desarrollo económico, aumentar el número de empleos, generalizar los beneficios de su desarrollo, y el crecimiento de las recetas fiscales del gobierno.

El desarrollo económico del país requiere que las decisiones de desarrollo del turismo de destino sean canalizadas ordenadamente y contribuyen al bienestar de población y al cumplimento de los objetivos del Plan del Buen vivir.

Existe una paradoja entre la necesidad de evaluar el comportamiento del consumidor y lo que se realiza en la actualidad, y la necesidad de considerar este comportamiento para lograr una gestión comercial de destinos turísticos exitosa. Se debe lograr:

1. Un control sistemático de los niveles de satisfacción del turista y que ello forme parte del criterio de evaluación, por encima del crecimiento en el número de visitantes;

2. Analizar de forma continua las reacciones de los residentes ante los turistas, pues la interacción residente-turista es un componente importante en la experiencia turística; y

3. Comprender que el desarrollo de las infraestructuras en el destino tiene implicaciones para los turistas que atrae, en los recursos medioambientales y en la población residente, condicionando el bienestar de turistas y residentes a largo plazo. 
A partir de tales aspectos, la gestión del desarrollo de los destinos turísticos en los territorios deberá tener un enfoque integrador, abarcando las distintas variables que inciden en los resultados y sobre las cuales el territorio tendrá más o menos posibilidades de accionar, de acuerdo con el desarrollo alcanzado y las propias condicionantes externas e internas. En los territorios deben realizarse acciones para gestionar el desarrollo considerando la importancia que en cada momento asumen las distintas variables.

\subsection{CONTRADICCIONES CULTURALES}

Según el programa del gobierno para la cultura es una de las principales fuerzas impulsoras de desarrollo integral, la misma constituye un elemento identitario, constructor de la unidad de la nación y factor de innovación tecnológica y de sustentabilidad, tanto en el aspecto social como en el económico.

Los proyectos se desarrollan al interior de las sociedades y organizaciones. En ellos participan personas que presentan comportamientos influenciados por el estilo y las costumbres. Por consiguiente, los resultados y la dinámica del proceso de evaluación del comportamiento del consumidor están constantemente afectada por la cultura. Las decisiones tomadas alrededor del mismo pueden ser vistas de diversas formas por los miembros de la sociedad y dependiendo de ello, asumirán posiciones contributivas, detractoras o imparciales sobre el proyecto de que se trate en el destino turístico.

\subsection{CONTRADICCIONES AMBIENTALES}

Para una mejor comprensión del papel de las organizaciones en la evaluación del comportamiento del consumidor ante la problemática social en su dimensión ambiental es necesario comprender, primeramente, el concepto de desarrollo sustentable. En la actualidad, cada vez más se habla en términos de crecimiento y desarrollo sustentable, es importante resaltar que el término crecimiento tiene un significado esencialmente material, en tanto el termino desarrollo sustentable es el proceso de mejora 
de las condiciones de vida de las comunidades y al mismo tiempo se refiere a los límites de capacidad de carga de los ecosistemas ${ }^{1}$.

El concepto de sustentabilidad tiene raíces en la ecología y está asociada a la capacidad de regeneración de los ecosistemas. Entretanto en la evaluación de proyectos, la problemática del medioambiente y del desarrollo se convierte en una discusión llena de conflicto y divergencias entre las partes interesadas. El criterio de sustentabilidad en el desarrollo del turismo confiere una gran importancia en la evaluación de los proyectos de inversiones al análisis de los impactos ambientales - ecológicos.

La satisfacción de las necesidades turísticas no debe constituir una amenaza para los intereses sociales y económicos de las poblaciones de las regiones turísticas, para el medio ambiente, especialmente para los recursos naturales, atracción esencial del turismo, ni para los lugares históricos-culturales.

Se puede entonces constatar la existencia de un conflicto entre el estímulo del desarrollo sustentable del turismo, con el consiguiente crecimiento de las inversiones y la posibilidad real de la perdida de los atractivos turísticos. Además el crecimiento en las inversiones destinadas a la creación de infraestructuras, construcciones y vías de comunicación de las que se beneficiará toda la población, al mismo tiempo, al no conferirse la importancia requerida a la temática medioambiental, se está produciendo una alteración irreversible en el paisaje y una seria afectación en el patrimonio natural.

La construcción masiva y el consiguiente uso indiscriminado de los recursos naturales, están provocando la huida de la fauna (principalmente marina), desaparición de especies vegetales muchas de las cuales constituyen fuente de alimentación de la población, además de provocar la acumulación de todo tipo de desperdicios.

1 Tomado de Bonkoski Buffara Lúcia Cristina, Desarrollo Sustentable y Responsabilidad Social (p. 27). 


\section{CONCLUSIONES}

En el proceso de evaluación del comportamiento del consumidor es necesario adoptar un enfoque integrado, que tenga en cuenta las dimensiones económica, social y ambiental para lograr una gestión comercial de destinos turísticos eficiente y eficaz.

Resulta necesario poner en mano de los actores y decidores un modelo evaluación que permita estandarizar el proceso de evaluación del consumidor, con flexibilidad, en correspondencia a las especificidades de cada producto turístico en el destino seleccionado.

Los resultados que se esperan obtener del desarrollo y aplicación del Modelo de evaluación del comportamiento del consumidor en la gestión comercial de destinos turísticos que se propone en esta investigación contribuirá a solucionar las fundamentales contradicciones presentadas. 


\section{REFERENCIAS BIBLIOGRÁFICAS}

Administración de empresas turísticas online. (s.f.). http://administracion.realmexico.info/2013/10/conceptosde-hotel.html

Aiteco Consultores. (s.f.). El Modelo SERVQUAL de Calidad de Servicio. https: / / www.aiteco.com/modeloservqual-de-calidad-de-servicio/

Antecedentes de la hotelería. (2007, 14 agosto). http://tecnologialbustamante.blogspot.com/

Antón, S., y Oliveras, J. (1998). Turismo y planificación del territorio en la España de fin de siglo. Universitat Rovira i Virgili.

Bigné, E., Font, X., y Andreu, L. (2001). Marketing de Destinos Turísticos: Análisis y etsrategias de desarrollo. ESIC Editorial.

CEGAHO Centro Empresarial Gastronómico Hotelero. (2013, 25 julio). Breve Historia de la Industria Hotelera. https://cegaho.wordpress.com/2013/07/25/historia-industria-hotelera/

Castillo, E. (2005). Escala Multidimensional SERVQUAL. https://www.gestiopolis.com/wp-content/ uploads/2010/03/mida-la-calidad-de-su-servicio-con-la-escala-servqual.pdf

Chávez, T. (2013, 4 diciembre). Índice de Satisfacción al Cliente (ISC). https://prezi.com/gzbtskpjqckf/ indice-de-satisfaccion-al-cliente-isc/

Comportamiento del consumidor. (2011, 27 abril). http://merk-behavior.blogspot.com/2011/04/capitulo-2marco-teorico-fig.html

Definición de servicio. (2011, 11 febrero). http://mejormarketing.blogspot.com/2011/02/definicion-deservicio.html 
DIASLEY. (2011, 4 octubre). Importancia del servicio al cliente. http://dana65773.blogspot.com/2011/10/ importancia-del-servicio-al-cliente.html

Ejarque, J. (2005). Destinos turísticos de éxito. Diseño, creación, gestión y marketing. Ediciones Pirámide.

Paul, J., y Olson, J. (2006). Comportamiento del consumidor y estrategia de marketing. McGraw-Hill.

Hawkins, D., Best, R., y Coney, K. (2004). Comportamiento del consumidor. Construyendo estrategias. McGraw-Hill.

Instituto Nacional de Estadísticas y Censo. (2010). Sistema Integrado de Consultas. (Ecuador en Cifras). Recuperado el 25 de julio de 2016, de: http://app.sni.gob.ec/sni-link/sni/Portal\%20SNI\%20 2014/FICHAS\%20F/1314_SUCRE_MANABI.pdf

Kotler, P., y Keller, K. (2006). Dirección de Marketing. Pearson Educación.

Kotler, P., y Armstrong, G. (2007). Marketing. Versión para Latinoamérica. Pearson Educación.

Kotler, P., y Armstrong, G. (2012). Marketing. Pearson Educación.

Kotler, P., Kartajaya, H., y Setiawan, I. (2010). Marketing 3.0. LID Editorial.

Lattin, G. W. (2012). Administración moderna de hoteles y moteles. Editorial Trillas.

Larraiza, L. (s.f.). Diferentes tipos de huesped. http://leirelarraiza.com/operativa/diferentes-tipos-dehuesped/

López, F., Marchena, M., Antón, S., Vera, J. F., y Vilà, J. (1997). Análisis territorial del turismo. Ariel Geografía.

Mármol, P., García, J., y Ojeda, G. (2016). Marketing Turistico. Ediciones Paraninfo. 
García, K. I., Huaman, J. G., Llanos, W. D., y Ticllacuri, F. M. (s.f.). Medición de la calidad de servicios. https://www.academia.edu/21604340/SERVQUAL_1_

Nava Uriostegui, J. M. (2013, 29 Octubre). Índice de satisfacción del cliente. http:/ /www.gestiopolis.com/ indice-de-satisfaccion-del-cliente/

Rodríguez, R. (1971). Modelos Matemáticos de Desarrollo Regional. Revista Economía y Desarrollo, 6.

Rodríguez, R. (1985). Planificación territorial de la economía socialista. EMPSES.

Rodríguez, R. (201 1). Destinos turísticos. Realidad y concepto. Revista de investigación en turismo y desarrollo local (TURyDES), 4(11). http://www.eumed.net/rev/turydes/11/rrf.html

Sandoval, G. (2012, 3 julio). Definición de hotelería. http://turismoholera.blogspot.com/2012/07/ definicion-de-hoteleria.html

Schiffman, L. G., y Kanuk, L. L. (2010). Comportamiento del Consumidor. Pearson Educación.

Thompson, I. (2019). La Satisfacción del Cliente. https://www.promonegocios.net/clientes/satisfaccioncliente.html

Vázquez, R., Díaz, A. M., y Suárez, L. (2007). Evaluación por los consumidores de sus experiencias de queja estrategias de recuperación del servicio y justicia percibida. Estudios sobre consumo, 81, 9-28. 